\title{
Dental Alterations in Sickle Cell Disease
}

\author{
Al-Jafar $\mathrm{H}^{*}$, Dashti $\mathrm{H}^{2}$, Al-Haddad $\mathrm{SJ}^{3}$, Al-qattan $\mathrm{S}^{4}$ and Al-Ramzi $\mathrm{A}^{5}$ \\ ${ }^{1}$ Consultant Hematologist - Amiri Hospital, Kuwait City, Kuwait \\ ${ }^{2}$ Assistant professor in Kuwait university, Dental College, Kuwait \\ ${ }^{3}$ Consultant in Orthodontric, Amiri Hospita - Dental Center, Kuwait \\ ${ }^{4}$ Registrar in Dental Primary Health Care, Adan Dental Center, Kuwait \\ ${ }^{5}$ Consultant, Maxillofascial surgeon, Bneid Al Gar Dentel Center, Kuwait
}

${ }^{*}$ Corresponding author: Al-Jafar H, Consultant Hematologist - Amiri Hospital, Kuwait City, Kuwait, E-mail: cbc9@hotmail.com

Citation: Al-Jafar H, Dashti H, Al-Haddad SJ, Al-qattan S, Al-Ramzi A (2016) Dental Alterations in Sickle Cell Disease. J Dent Oral Care Med 2(2): 203. doi: 10.15744/2454-3276.2.203

Received Date: February 25, 2016 Accepted Date: May 11, 2016 Published Date: May 13, 2016

\begin{abstract}
Sickle cell disease is a congenital blood disorder. The abnormal hemoglobin causes microinfarcts that lead to multi-organ alterations, including dental involvement. The entire oral and maxillofacial region may be involved, affecting the teeth, multiple oral structures, and maxillofacial bones. Dental complications include delayed eruption, tooth malformation, dental caries, teeth discoloration, and pulp necrosis. Oral soft tissue complications can involve the tongue, gingiva, and oral mucosa. Dental complications of the oral and maxillofacial bones include anatomical, radiographical, and structural changes. Vaso-occlusion is a major factor in sickle cell disease, which induces multiple dental complications. Oral, fascio-maxillary, and dental complications depend not only on the presence of sickle cell disease, but also on factors related to the patient's personal oral medications, social life, and habits such as diet and oral hygiene.

Keywords: Sickle cell disease; Dental complications; Dental caries; oral hygiene
\end{abstract}

\section{Introduction}

Sickle cell disease (SCD) occurs due to a genetic mutation that results in amino acid substitution of valine to glutamic acid at position 6 of the adult B-globin chain. In the homozygous form, the substitution results in polymerization of hemoglobin on deoxygenation, leading to deformed dense red blood cells in patients with SCD. The predominant pathophysiological feature of SCD is vaso-occlusion, which leads to acute and chronic complications such as: painful crises; increased risk of infection; acute chest syndrome; and stroke. It may also cause severe painful episodes with dramatic hemolysis that can progress to involve multiple organs; including teeth, bones, lungs, liver, kidneys central nervous system, and cardiovascular system [1,2]. In SCD patients, dental care is not considered a priority in comparison to other major complications such as recurrent severe vasoocclusive painful crises, chest infection, liver, renal and many other life threatening complications. In this article, we focused on dental complications in SCD patients, which require specialized dental care and awareness among dental practitioners. Three major alterations are going to be discussed in this article: dental, oral soft tissues, and maxillofacial complications (Figure 1) [3].

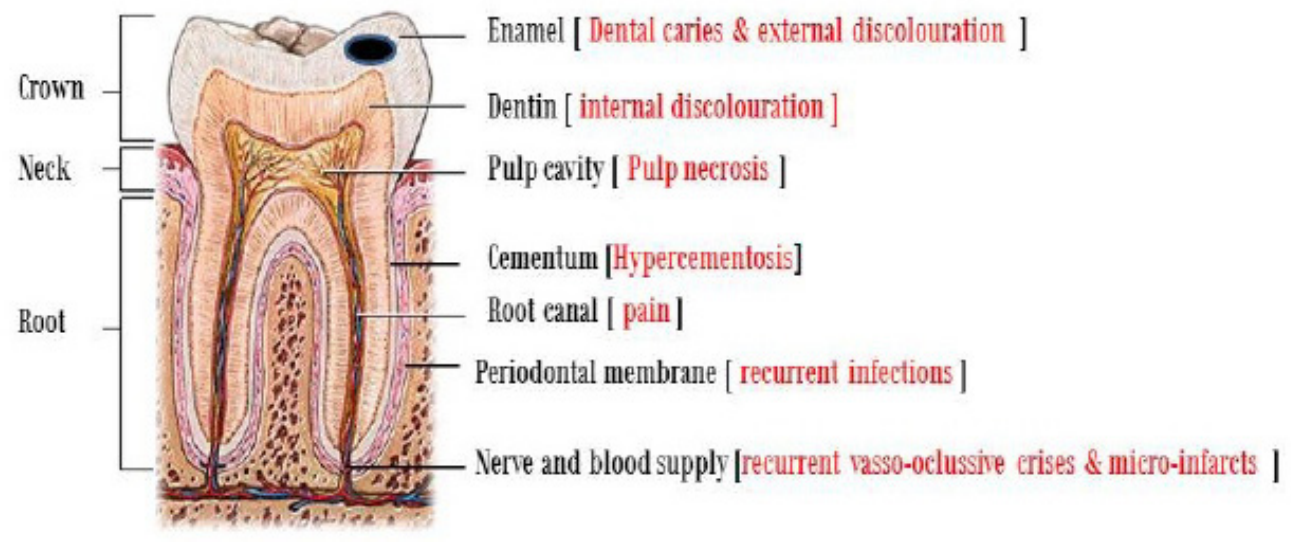

Figure 1: Dental anatomy and main complications in sick cell disease 


\section{Dental Alterations}

\section{Eruption}

Tooth eruption is defined as the continuous biological process by which developing teeth emerge through the jaw and the overlying mucosa to enter the oral cavity and contact teeth in the opposing arch. Both primary and permanent teeth follow a specific sequence of eruption. Genetics, hormones, geographical location, ethnicity, sex, economic status, nutrition, and growth are all factors that influence tooth eruption [4]. Delayed eruption of teeth has been reported in SCD patients, where disturbances in growth and development such as ischemic infarctions of the maxilla and mandible play a major role in such anomalies [5]. In a cross-sectional study done by Mendes, et al., SCD patients were found to have a 1.7 times greater chance of delayed eruption in comparison to healthy individuals [6].

\section{Tooth formation}

Abnormal tooth formation has been documented in SCD [7]. Anatomically, teeth are divided into four structures: enamel, dentin, cementum, and the pulp [8]. The first three are hard, non-viable, calcified structures, while the pulp consists of soft viable structures such as blood and lymphatic vessels, nerves, fibroblasts, and collagen fibers [9]. Enamel formation undergoes three steps (a) matrix formation: where enamel proteins are laid down by ameloblasts, (b) mineralization: where minerals are deposited and enamel proteins are removed, (c) maturation: further mineralization and protein removal. Dentin and cementum formation tend to follow the same steps. Any disturbances during tooth formation, whether local or systemic, can result in permanent tooth defects [10]. Enamel and dentin hypomineralization and hypomaturation have been reported in SCD patients [11]. Interestingly, such abnormalities were observed in both heterozygous and homozygous states of sickle cell disorders [12]. The maxillary and mandibular bone marrows attempt to compensate for the low numbers of red blood cells by becoming hyperplastic, and cause jaw expansion. Such compensatory hematopoietic expansion is believed to induce changes in dental tissues in general [13]. Hypercementosis also has been associated with SCD, where excessive deposition of non-neoplastic cementum is layed over the root's primary cementum [14]. Such phenomenon alters the root shape and morphology. The cellular cementum resembles bone and is named osteo-cementum [15].

\section{Dental caries}

Due to hypomineralization and hypomaturation of teeth in SCD patients, tooth structure is at higher risk of developing dental caries [16]. In addition, the continuous use of sucrose-based medications and frequent hospitalization would result in improper oral hygiene [17]. A study was conducted comparing 35 dental patients with SCD, aged 6 years and older, to 140 dental patients without SCD (age-gender-matched study) to determine whether individuals with SCD had higher prevalence of dental caries using the Decayed, Missing, and Filled Teeth Index (DMFS index). The mean DMFS scores for subjects aged 18 and older were found $29 \%$ higher in patients with SCD. In addition, the average number of infected teeth surfaces for all subjects with SCD was $41 \%$ greater than the subjects without SCD [3]. In a cross-secional study by Fernandes, et al. in Brazil, young children (average age 8.9 years) with SCD were found to have low experience of dental caries in comparison to healthy individuals at the same age [18]. On the other hand Singh, et al. examined 750 patients ranging in age from 3-15 years for their oral and periodontal status. Patients were divided into 3 groups: the first group included 250 patients diagnosed with Thalassemia major, the second group included 250 diagnosed with SCD, and the third group included 250 healthy individuals as controls. While comparing DMFT results between the second and third groups, Singh found a significant difference, where in SCD patients experienced higher caries rates [19].

\section{Tooth discoloration}

SCD patients can present with tooth discoloration, where teeth appear abnormal in comparison to the normal range of variation in color and shade of the teeth [20]. Tooth discoloration is divided into intrinsic and extrinsic discoloration; the former occurs during tooth development due to endogenous materials that have been incorporated into the dentin and enamel such as the severe hyperbilirubinemia, while the later develops after eruption due to exogenous pigments. Intrinsic staining of teeth can occur even after tooth development and eruption due to prolonged exposure to different types of pigments, where stains are incorporated into the enamel and dentin. In SCD, smoking, medications, and particular foods could induce micro-infarcts, which can be entrapped within the dentinal tubules and cause pre-eruptive red staining [21,22]. Another explanation for SCD-related tooth discoloration is the release of hemoglobin and its byproducts due to erythrocytosis into the peripheral circulation where they incorporate into the dentin and enamel and cause intrinsic discoloration [20].

\section{Pulp necrosis}

Asymptomatic pulp necrosis was reported in higher frequency among SCD patients than healthy people. Such a phenomenon was attributed to abnormal blood flow to the dental pulp [23]. Cox and Suni analyzed tooth sections from SCD patients and healthy individuals. He found sickle-shaped cells in the pulpal blood vessels of SCD patients 2-3 days after the sickle cell acute crisis. They concluded that would induce infarction/thrombosis of dental pulp vessels and eventually lead to pulpal hypoxia then necrosis and 
pathologic periapical abnormalities [24]. Pulpal pain observed in SCD patients is attributed to pulpal hypoxia and abnormal blood flow, where other common factors are absent, such as caries, traumas, cysts, and tumors. In addition, thrombosis is considered the primary factor associated with dental pulp necrosis in such patients [23].

\section{Oral soft tissue Alterations}

\section{Tongue}

Depapillation of the tongue, atrophic glossitis, is observed in anemic patients, while the frequency of such features is undetermined among SCD patients. Patients diagnosed with pernicious anemia, iron deficiency anemia, and SCD have expressed such clinical findings. Atrophic tongue is attributed to iron deficiency and vitamine B12 deficiency in SCD $[25,26]$. The tongue appears "beefy red" due to the loss of the filiform and fungiform papillae. The surface area of the depapillated tongue is related to the severity of the anemic condition. Furthermore, patients might complain of altered taste and dysphagia [13].

\section{Gingiva}

Although many researchers have considered the relationship between gingival/periodontal disease and SCD, studies in this field have been unable to obtain equivalent results. Several studies have indicated that SCD patients tend to express high plaque scores and subsequently develop gingivitis [27]. As opposed to adults, children with SCD are more prone to develop periodontitis. In general, SCD patients are more susceptible to infections and periodontal disease [17]. Gingival hypertrophy has also been observed in SCD patients [28]. Many studies have documented the expression of inducible nitric oxide synthase (iNOS) due to chronically inflamed gingiva related to infection [27]. On the other hand, a cross-sectional study by Passos, et al. concluded that risk factors such as older age and absence of daily use of dental floss were major causes of periodontal disease rather than a direct effect of SCD [29]. Olivieri, et al. have also indicated no significant difference between SCD patients and healthy individuals in regards to periodontal disease [30].

\section{Oral mucosa}

The oral mucosa in SCD patients presents with pallor to yellow discoloration, which is the result of deposition of blood pigments secondary to hyperbilirubinemia caused by erythrocytosis [7]. In addition, atrophy and recurrent aphthous ulcerations have been observed. Furthermore, erythematous macular lesions, bleeding, and hemorrhage can involve the buccal and labial mucosa. Histologically, it has been proven that buccal mucosal cells in SCD exhibit an increased number of enlarged nuclei [13]. Some investigators suggested a hypothesis to explain the observed atrophy of oral mucosa; they have suggested that keratinocytes exhibit abnormal maturation process and exfoliate prematurely before the reduction in the nuclear size [25].

\section{Maxillofacial Alterations}

\section{Bone anatomical and radiographic dental changes}

SCD patients are commonly affected by bone and joint complications. The most common area of bone destruction is the femoral head, but other regions have also been reported, such as the humeral head, thoracic and lumbar spine, and temporomandibular joint (TMJ). A few papers in the literature have described TMJ disorders in SCD patients; unfortunately all have been clinical case reports. In a reported clinical case of a 23-year-old woman with SCD, early signs of bone infarction of the humeral head and avascular necrosis of the TMJ and femur head have been observed. Interestingly, all were located on the left side. These abnormalities were diagnosed by clinical examination and computed tomography. In another case, TMJ disorder was reported in a 23-year-old woman with SCD, who presented with no history of trauma or orthognathic surgery. As a potential complication mouth opening, central line deviation for the right side, and slight facial asymmetry because of the short right ramus height. Furthermore, flattened condylar head and avascular necrosis of glenoid fossa noted in the right joint through radiographic examination and computed tomography. Caracas, et al. reported a rare case of aseptic arthritis in the TMJ of a 22 -year-old woman with SCD. The patient in this situation developed pain in the left preauricular region, hyperalgesia of the left masseter muscle, mouth opening limitation, and mild edema [31].

Due to compensatory hematopoiesis, hemolysis, and vaso-occlusive events in the maxilla and mandible, radiographic bone changes are noticed in SCD patients [32]. These changes include (a) increased radiolucency which leads to decreased trabeculae numbers, (b) increased medullary spaces, (c) thinning of the inferior mandibular border, (d) staircase pattern noticed mainly in the alveolar interproximal bone due to horizontal trabecular rows, (e) thickening of the lamina dura, (f) resorption in alveolar bone, and (g) radiopacities due to repaired mechanism of bony infarctions [33]. Pain in the jaws is common due to vaso-occlusion, which might lead to acute and chronic ischemia and infarction followed by osteonecrosis [34]. Vaso-occlusion could also cause anesthesia or paresthesia of the mandibular nerve [35]. Hypovascularity in the mandible can induce mandibular osteomyelitis and osteonecrosis in SCD patients. Mandibular osteomyelitis can be followed by osteosclerosis if proper healing is achieved [13].

Disturbances of growth and development could occur among SCD patients, which lead to dentofacial deformities such as malocclusion [3]. In addition, craniofacial anomalies are associated with SCD and the most common bone abnormalities reported in the literature are maxillary protrusion and mandibular retrusion, which subsequently result in overjet, overbite, and spacing of 
both dental arches [36]. Malocclusion in its different classes can be observed in SCD patients due to bony changes. Class I malocclusion is the most prevalent form of malocclusions among SCD patients, even more prevalent than normal occlusion, and there is no clear consensus concerning its etiology. Individuals with Class I malocclusion have normal molar relationships, but their teeth are not in the correct position in the line of occlusion due to mal-opposed teeth, rotation, spacing, overbites, open bites, posterior cross bites, or even anterior crossbites [37].

Class II malocclusion is characterized into two groups, skeletal and dental. Skeletal class II malocclusions are jaw-related, where dental class II malocclusions are dentoalveolar related; however, a combination of skeletal and dental Class II malocclusions is observed in most SCD cases. This malocclusion is related to retrognathic mandible, prognathic maxilla, or a combination of both. Skeletal Class II malocclusion is most frequent in particular clinics, caused mainly by retrognathic mandible [38]. Malocclusion, especially severe class II malocclusion, would be an inevitable result in SCD patients, leading to chewing, swallowing, phonation and breathing disorders, and even psychosocial disorders, where the patients' self-esteem and interpersonal relationships might be severely affected [36]. Orofacial complications depend not only on the presence of SCD but on other factors that are related to the subjects themselves, such as: oral hygiene, dietery habits, and social condition [23].

\section{Prevention of dental complications in SCD}

SCD patients need to avoid sickle cell precipitating factors such as extreme hot and cold weather, low oxygen pressure atmosphere, severe muscular exercise to avoid vaso-occlusive crises. They also must recognize the need to take their painkillers and antibiotics whenever required in their frequent sickle cell crises [39]. Such patients require regular dental checkups to detect early dental abnormalities or maxillary osteonecrosis to be treated before major dental complications develop. The treatment of choice consists of folic acid, hydroxocobalamin, mouth wash, rehydration, opioid analgesics and non-steroidal anti-inflammatory drugs to manage the severe pain and reduce inflammation, or even a blood transfusion to reduce the anemic crisis[40]. Health professionals should be aware that SCD may also provoke aseptic jaw arthritis.

\section{Conclusion}

Dental and Bone complications in SCD patients due to recurrent micro-infarctions are serious complications. It affects both jaws and subsequently the teeth, causing multiple malformations, which in turn, affect teeth function and integrity. Different approaches need to be used in these patients to preserve healthy dental status: excellent oral hygiene, regular follow-up, compliance with treatment, and early orthodontic treatment if required. All these parameters could significantly affect patients' quality of life when taking into consideration dental, oral, and maxillofacial health status.

\section{References}

1.Hiran S (2005) Multiorgan dysfunction syndrome in sickle cell disease. J Assoc Physicians India 53: 19-22.

2. Mousa SA, Al Momen A, Al Sayegh F, Al Jaouni S, Nasrullah Z, et al. (2010) Management of painful vaso-occlusive crisis of sickle-cell anemia: consensus opinion. Clin Appl Thromb Hemost 16: 365-76.

3. Laurence B, George D, Woods D, Shosanya A, Katz RV, et al. (2006) The association between sickle cell disease and dental caries in African Americans. Spec Care Dentist 26: 95-100.

4. Kutesa A, Nkamba EM, Muwazi L, Buwembo W, Rwenyonyi CM (2013) Weight, height and eruption times of permanent teeth of children aged 4-15 years in Kampala, Uganda. BMC Oral Health 13: 15.

5. Erdogan O, Kisa H, Charudilaka S (2011) Sickle-cell disease: a review of oral manifestation and presentation of a case with an uncommon complication of the disease. Rangsit J Arts Sci 3: 179-85.

6. Mendes PH, Fonseca NG, Martelli DR, Bonan PR, de Almeida LK, et al. (2011) Orofacial manifestations in patients with sickle cell anemia. Quintessence Int 42: 701-9.

7. Mello SM, Paulo C Araujo R, Alves C. (2012) Oral considerations in the management of sickle cell disease: a case report. Oral Health Dent Manag 11: 125-8.

8. Metivier A, Bland K (2014) Dental Anatomy: A Review. Continuing Dental Education.

9. Pashley DH, Walton RE, Slavkin HC (2002) Histology and Physiology of The Dental Pulp. Endodontics 25-61.

10. Wallman SC, Jenssen L, Tran HQ (2011) Classification of severe tooth discolorations and treatment options. 1-49.

11. Pithon MM (2011) Orthodontic treatment in a patient with sickle cell anemia Am J Orthod Dentofacial Orthop 140: 713-9.

12. Adewoyin AS (2015) Management of sickle cell disease: a review for physician education in Nigeria (sub-saharan Africa). Anemia 791498.

13. DeRossi S, Garfunkel, A, Greenberg M (2003) Hematologic Diseases. In M Greenburg, M Glick Burket's oral medicine: diagnosis and treatment 10th ed, Hamilton, Canada: BC Decker Inc 429-53.

14. Chaudhary M, Agarwal R, Holani A (2012) Histological changes in tooth enamel, dentin and cementum of patients with sickle cell anemia. J Oral Health Res 3: 1-4.

15. Pappen F G, Fagonde CD, Martos J, Silveira LFM (2011) Hypercementosis: a challenge for endodontic therapy. South Brazilian Dentistry RSBO 8: 321-8.

16. Mehrotra V, Sawhny A, Gupta I, Gupta R (2014) Tell tale shades of discoloured teeth: a review. Indian J Dental Sci 6: 95-9.

17. Luna ACAE, Rodrigues MJ, Menezes VA, Marques KMG, Santos FA Dos (2012) Caries prevalence and socioeconomic factors in children with sickle cell anemia. Braz Oral Res 26: 43-9.

18. Fernandes MLMF, Kawachi I, Corrêa-Faria P, Pattusi MP, Paiva SM, et al. (2015) Caries prevalence and impact on oral health-related quality of life in children with sickle cell disease: cross-sectional study. BMC Oral Health 15: 68. 
19. Singh J, Singh N, Kumar A, Kedia NB, Agarwal A (2013) Dental and periodontal health status of Beta thalassemia major and sickle cell anemic patients: a comparative study. J Int Oral Health 5: 53-8.

20.Walsh LJ, Liu JY, Verheyen P (2004) Tooth Discolouration and Its Treatment Using KTP Laser-assisted Tooth Whitening. J Oral Laser App 4: 7-21.

21. Sulieman M (2005) An overview of tooth discoloration: extrinsic, intrinsic and internalized stains. Dental Update 32: $463-8,471$.

22. Plotino G, Buono L, Grande NM, Pameijer CH, Somma F (2008) Nonvital Tooth Bleaching: A Review of the Literature and Clinical Procedures. J Endod 34: 394-407.

23. Javed F, Correa FO, Nooh N, Almas K, Romanos GE, et al. (2013) Orofacial manifestations in patients with sickle cell disease. Am J Med Sci 345: $234-7$.

24. Cox GM, Soni NN (1984) Pathological effects of sickle cell anemia on the pulp. ASDC J Dent Child 51: 128-32.

25. Paraizo JU, Rech IA, Azevedo-Alanis LR, Pianovski MA, De Lima AA, et al. (2013) Cytomorphometric and cytomorphologic analysis of oral mucosa in children with sickle cell anemia. J Cytol 30: 104-8.

26. Graells J, Ojeda RM, Muniesa C, Gonzalez J, Saavedra J (2009) Glossitis with linear lesions: an early sign of vitamin B12 deficiency. J Am Acad Dermatol 60: 498-500.

27. Guzeldemir E, Toygar HU, Boga C, Cilasun U (2011) Dental and periodontal health status of subjects with sickle cell disease. J Dent Sci 6: 227-34.

28. Sambandan T (2010) Review on Oral Manifestations of Blood Diseases. J Indian Academy of Dental Specialist 41: 41-3.

29. Passos CP, Santos PR, Aguiar MC, Cangussu MC, Toralles MB, et al. (2012) Dental condition in sickle cell disease Sickle cell disease does not predispose to caries or periodontal disease. Spec Care Dentist 2: 55-60.

30. Olivieri NF, Pakbaz Z, Vichinsky E (2011) Hb E/beta-thalassaemia: a common \& clinically diverse disorder. Indian J Med Res 134: 522-31.

31. Santos B, Dioné P, Machado S, Passos CP, Aguiar MC, et al. (2013) Prevalence of orofacial alterations in sickle cell disease : a review of literature. Braz J Oral Scl 12: 153-7.

32. Saito N, Nadgir RN, Flower EN, Sakai O (2010) Clinical and radiologic manifestations of sickle cell disease in the head and neck. Radiographics 30: 1021-34. 33. Sams DR, Thornton JB, Amamoo PA (1990) Managing the dental patient with sickle cell anemia : a review of the literature. Pediatr Dent 12: $316-20$.

34. da Silva Junior GB, Daher Ede F, da Rocha FA (2012) Osteoarticular involvement in sickle cell disease. Rev Bras Hematol Hemoter 34: 156-64.

35. Neville B, Damm D, Allen C, Bouquet J (2009) Hematologic Disorders. In Oral and Maxillofacial Pathology 3rd ed, Elsevier 571-612.

36. Costa CP, de Carvalho HL, Thomaz EB, Sousa Sde F (2012) Craniofacial bone abnormalities and malocclusion in individuals with sickle cell anemia: a critical review of the literature. Rev Bras Hematol Hemoter 34: 60-3.

37. Buschang PH (2014) Class I malocclusions-The development and etiology of mandibular malalignments. Seminars in Orthodontics 20: 3-15.

38. Freitas MR, Santos MA, Freitas KM, Janson G, Freitas DS, et al. (2005) Cephalometric characterization of skeletal Class II, division 1 malocclusion in white Brazilian subjects. J Appl Oral Sci 13: 198-203.

39. Atherley G, Taylor L, Powell E (2010) College of Dental Hygienists of Ontario CHDO Advisory Sick Cell Diseases, 2008, 2009, 2010, Identified In The Dental Hygiene Act 1991- O. Reg. 219/94 Part III.

40. Guy's and St. Thomas' NHS Foundation Trust @ 2010 Pediatric Formulary $9^{\text {th }}$ Edition Revised December 2012.

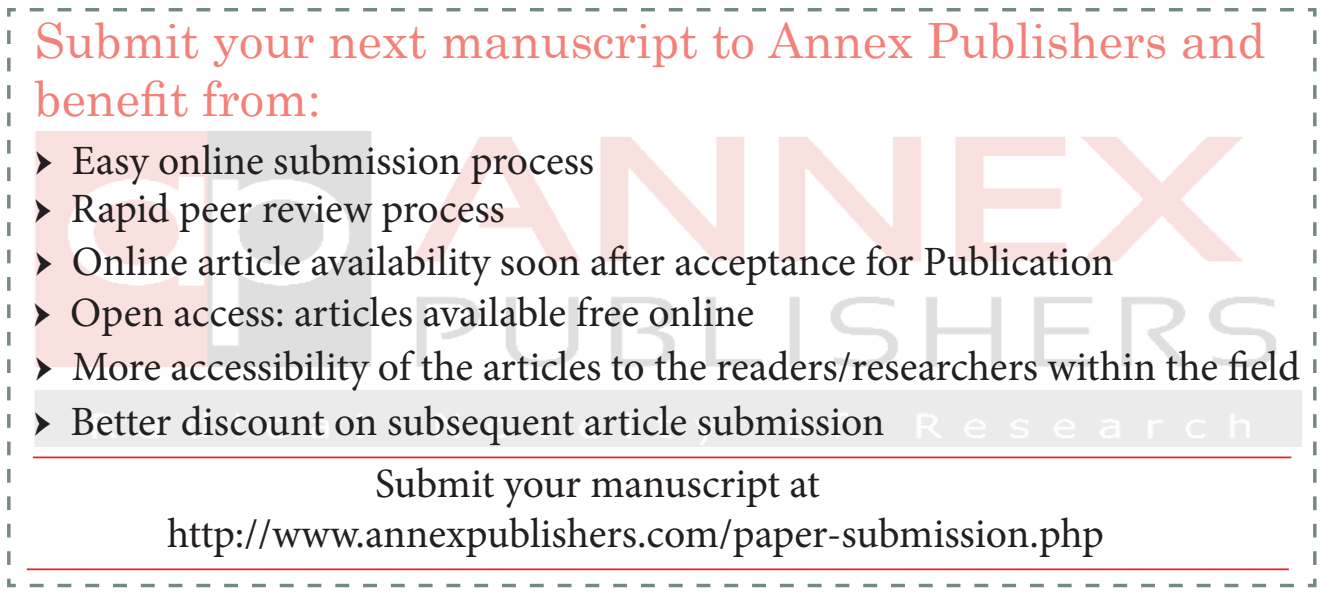

